\title{
Fabrication, characterization, and application- matched design of thermoelectric modules based on Half-Heusler FeNbSb and TiNiSn
}

Cite as: J. Appl. Phys. 126, 085113 (2019); doi: 10.1063/1.5108636

Submitted: 2 May 2019 . Accepted: 6 August 2019.

Published Online: 27 August 2019

Daniel Landmann, Yinglu Tang, Benjamin Kunz, Renato Huber, Daniel Widner, Peter Rickhaus,

Remo N. Widmer, (D) Hans Rudolf Elsener, and Corsin Battaglia ${ }^{a)}$

\author{
AFFILIATIONS \\ Empa, Swiss Federal Laboratories for Materials Science and Technology, 8600 Dübendorf, Switzerland
}

Note: This paper is part of the special topic on Advanced Thermoelectrics.

a) Author to whom correspondence should be addressed: corsin.battaglia@empa.ch

\begin{abstract}
We describe the fabrication of thermoelectric modules based on Half-Heusler TiNiSn and FeNbSb and their performance assessment under different boundary conditions. Module design is guided by a multiphysics model implementing experimentally determined thermoelectric materials properties. We consider two types of boundary conditions: first, imposing fixed cold- and hot-side temperatures onto the module, and second, imposing fixed values for the heat transfer coefficients between module and heat sink and source, representative for a waste-heat-recovery system using the exhaust heat of an internal combustion engine. We compare the modeling results with experimental data obtained from Half-Heusler modules integrated into a heat exchanger mounted to the exhaust of a compact van.
\end{abstract}

\section{Published under license by AIP Publishing. https://doi.org/10.1063/1.5108636}

\section{INTRODUCTION}

Thermoelectric materials enable the conversion of heat into electric power and have the potential to improve the energy efficiency of vehicles with internal combustion engines through waste-heat recovery. Intensive research efforts have focused on understanding and optimizing thermoelectric material properties, thus improving the thermoelectric figure of merit $z T,^{1-13}$ but only a few attempts to build thermoelectric modules and assess their performance under conditions relevant to vehicle operation are reported in the literature. ${ }^{14-20}$ In addition, thermoelectric modules are often tested on thermoelectric test benches imposing constant cold- and hot-side temperatures. These boundary conditions are only relevant in rare application cases and are not representative of a waste-heat-recovery system in a vehicle.

Here, we describe the fabrication of thermoelectric modules based on Half-Heusler TiNiSn and FeNbSb. Module geometry is guided by multiphysics modeling to maximize electrical power output by matching the thermal resistance of the module to the hotand cold-side heat exchanger of a vehicle waste-heat-recovery system.

Experimental module output data, including data from a waste-heat-recovery system operated on a compact van under steady-state conditions, are compared to the modeling results.

\section{EXPERIMENTAL SECTION}

Arc melting in vacuum was used to prepare ingots of $p$-type $\mathrm{FeTi}_{0.2} \mathrm{Nb}_{0.8} \mathrm{Sb}$ (abbreviated as FeNbSb)and n-type $\mathrm{TiNi}_{1.06} \mathrm{Sn}_{0.98}$ (abbreviated as TiNiSn) Half-Heusler alloys from the elements (for more details, see Ref. 7). Ingots were crushed and ground to powder with pestle and mortar, sieved to below $0.5 \mathrm{~mm}$, and milled in a planetary ball mill in an argon atmosphere at $600 \mathrm{rpm}$ for 15 cycles of $2 \mathrm{~min}$ with a break of $2 \mathrm{~min}$ in between each cycle [Fig. 1(a)]. $70 \mathrm{~g}$ of finely milled Half-Heusler powder was transferred in argon into a carbon die with an inner diameter of $50 \mathrm{~mm}$ and consolidated using spark plasma sintering between two $0.25 \mathrm{~mm}$ thick nickel discs with a diameter of $50 \mathrm{~mm}$. To improve the mechanical and electrical contact between the p- and n-type Half-Heusler material and the nickel disc, $2 \mathrm{~g}$ of $\mathrm{TiH}_{2}$ powder was mixed with $2 \mathrm{~g}$ of Half-Heusler powder and filled into the die between both sides of the bulk Half-Heusler powder and the neighboring nickel discs before sintering. This method reduces the average module contact resistance to below $1 \mathrm{~m} \Omega$. Spark plasma sintering was performed at a pressure of $50 \mathrm{MPa}$ in the argon atmosphere by heating the $p$-type and $n$-type material up to $1000^{\circ} \mathrm{C}$ and $900{ }^{\circ} \mathrm{C}$, respectively, within $10 \mathrm{~min}$, followed by a 
a)

b)
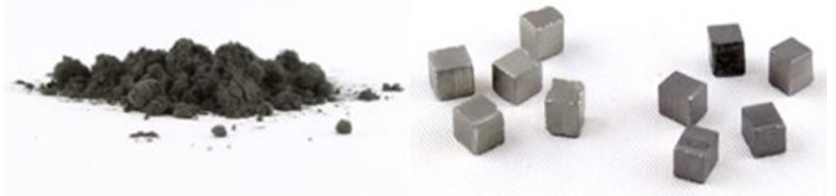

c)
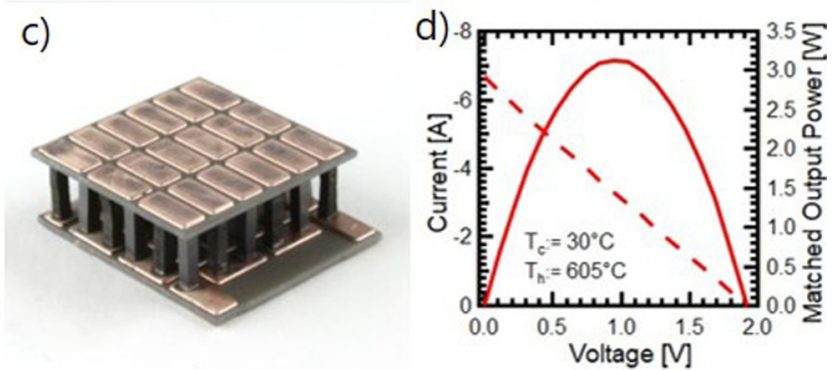

FIG. 1. (a) Half-Heusler powder after milling, (b) Half-Heusler legs with nickel contacts, (c) Half-Heusler module consisting of 18 p-type and 18 n-type legs connected electrically in series between two ceramic aluminum nitride plates with copper contacts, and (d) current-voltage and power-voltage characteristics of Half-Heusler thermoelectric module measured at $30^{\circ} \mathrm{C}$ cold-side temperature and $605^{\circ} \mathrm{C}$ hot-side temperature.

temperature hold of $10 \mathrm{~min}$ and $5 \mathrm{~min}$, respectively, and subsequent passive cooling down to room temperature. Using a diamond wire saw, the sintered pellets were cut into individual legs [Fig. 1(b)] with a base area of $1.75 \times 1.75 \mathrm{~mm}^{2}$ and a height of $4.0 \mathrm{~mm}$ (controlled by the amount of powder filled into the die). Thermoelectric modules [Fig. 1(c)] were then assembled by brazing an array of Half-Heusler legs onto two $0.6 \mathrm{~mm}$ thick ceramic aluminum nitride plates with a base area of $25 \times 25 \mathrm{~mm}^{2}$. Direct-bonded-copper contact layers with $0.3 \mathrm{~mm}$ thickness on the aluminum nitride plates connect $p$-type and $n$-type legs electrically in series and thermally in parallel. The brazing material is a standard nickel brazing material consisting of $74 \%$ nickel, $14 \%$ chromium, $4.5 \%$ iron, $3 \%$ boron, and $4.5 \%$ silicon. Brazing of the module assembly including the ceramic aluminum nitride plates with copper contacts was performed in a spark plasma sintering furnace at $4 \mathrm{kN}$ in the argon atmosphere by heating to $900{ }^{\circ} \mathrm{C}$ within $30 \mathrm{~min}$, followed by a temperature hold of $1 \mathrm{~min}$, and passive cooling down to room temperature. During brazing, current flows through the resistive carbon die, heating the die. By thermal conduction, the module assembly is also heated. Two $1 \mathrm{~mm}$ thick copper wires were then soldered directly onto the copper plates to measure the current-voltage characteristics of the modules [Fig. 1(d)] in vacuum with a source meter. The test bench is equipped with a resistive heater consisting of tantalum wires embedded into an aluminum nitride ceramic and a heat sink consisting of a water-cooled copper block. Hot- and cold-side temperatures were measured with $0.75 \mathrm{~mm}$ type $\mathrm{K}$ thermocouples embedded at the contact pad of the module.
Phase purity of the $p$-type and $n$-type Half-Heusler materials was confirmed by X-ray diffraction. Seebeck coefficient $(S)$ and electrical conductivity $(\sigma)$ were measured under the argon atmosphere. Thermal conductivity $(\kappa)$ of the thermoelectric material was calculated using $\kappa=\rho \alpha C_{p}$, with the thermal diffusivity $(\alpha)$ measured along the cross-plane direction by the laser flash method under argon flow without the contacting layers. The density $\rho$ of the samples was determined also without the contacting layers by determining the volume geometrically. The specific heat capacity $C_{p}$ was determined using the Dulong-Petit law $C_{p}=3 k_{B}$ per atom of the unit cell. See Ref. 7 for more details on the materials characterization.

Multiphysics modeling was performed within a COMSOL environment. The steady-state model incorporates the exact geometric details of the module and experimentally determined physical properties for the thermoelectric materials (Fig. 2) and the contact layer sequence in the contacts (as shown in Fig. S2 in the supplementary material). Solving the equation for heat and charge conservation yields the temperature and current for each computational node. For rapid screening through geometric and physical property spaces, the model is fully parametrized. The module is embedded into a virtual test bench, which can either impose constant cold- and hot-side temperature onto the module or constant heat transfer coefficients between the module and heat sink and source. a)

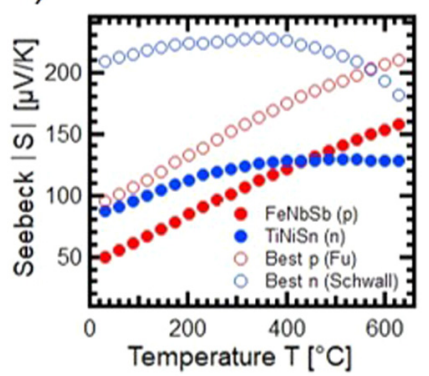

c)

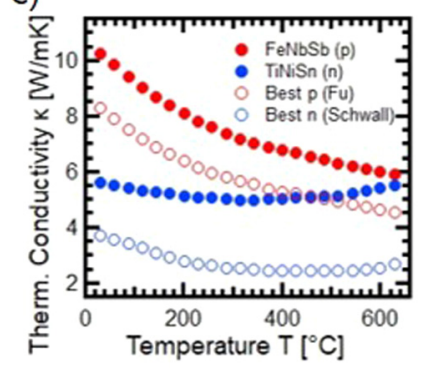

b)

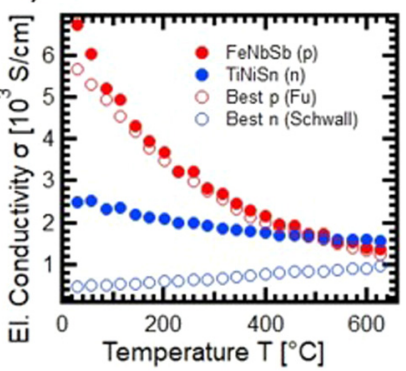

d)

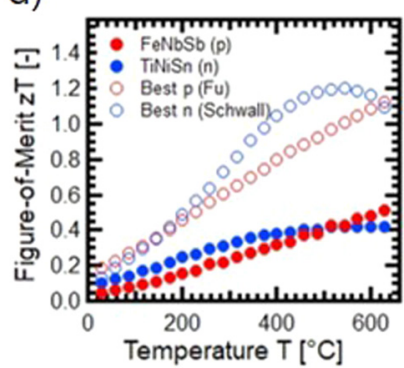

FIG. 2. Temperature dependence of (a) Seebeck coefficient, (b) electronic conductivity, (c) thermal conductivity, and (d) thermoelectric figure of merit of the p-type and n-type Half-Heusler materials used to build the thermoelectric modules and for the multiphysics modeling. Values for best-in-class p-type and n-type Half-Heusler materials from Refs. 13 and 21 are also shown for comparison. 


\section{RESULTS AND DISCUSSION}

Figure 2 shows the temperature dependence of the Seebeck coefficient $\mathrm{S}$ [plotted as $|\mathrm{S}|$ in Fig. 2(a), $\mathrm{S}<0$ for $\mathrm{n}$-type material, $S>0$ for p-type material], electronic conductivity $\sigma$, thermal conductivity $\kappa$, and thermoelectric figure of merit $z T$ of the p-type FeNbSb and n-type TiNiSn materials used to build the thermoelectric modules. Also shown in Fig. 2 are the corresponding values for p-type $\left(\mathrm{FeNb}_{0.88} \mathrm{Hf}_{0.12} \mathrm{Sb}\right)$ and $\mathrm{n}$-type $\left(\mathrm{Ti}_{0.5} \mathrm{Zr}_{0.25} \mathrm{Hf}_{0.25} \mathrm{NiSn}_{0.98} \mathrm{Sb}_{0.002}\right)$ Half-Heuslers reported in Ref. 13 [Figs. 2(a) and 3(a)-3(c)] and Ref. 21 [Figs. 3 and 5] with some of the highest reported peak $z T$ values for Half-Heusler materials in the literature. Based on the figure of merit $z T$, a theoretical upper limit for the module efficiency can be estimated. ${ }^{22}$ Generally, the efficiency of a thermoelectric module is defined by the ratio of the electrical power output divided by the thermal power supplied. ${ }^{22}$ In this work, we concentrate on maximizing the power output of the modules and not their efficiency. We note that our materials reported here are synthesized and processed in relatively large quantities to generate a baseline result and enable module development. There is still much room for improving the processing parameters to enhance the thermoelectric material properties. Also note that the nominal composition of our $p$-type FeNbSb material is comparable to the p-type material reported in Ref. 13, while our $n$-type TiNiSn material is free of expensive hafnium employed in Ref. 21. The low thermal a)

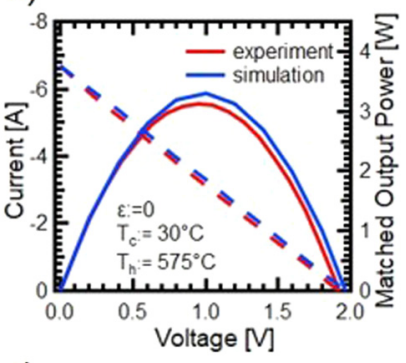

c)

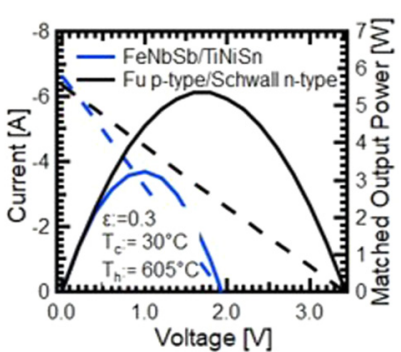

b)

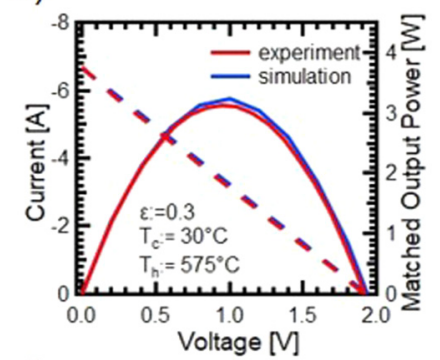

d)

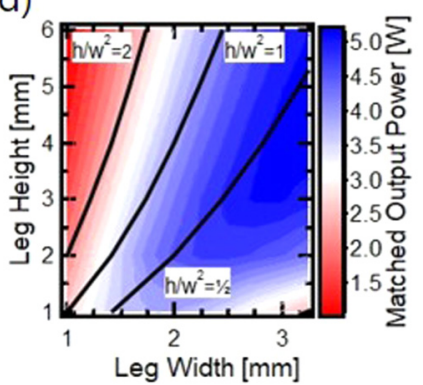

FIG. 3. Comparison of measured and simulated module output data (a) without and (b) with taking into account radiative heat losses in the model, (c) comparison of simulated module output data for the Half-Heusler material used to fabricate the modules and best-in-class Half-Heusler materials reported in Refs. 13 and 21, (d) module output power as a function of leg width (w) and leg height (h) at fixed cold- and hot-side temperatures with lines of constant $h / w^{2}$ ratio (given in units of $1 / \mathrm{mm}$ ). conductivity of the material in Ref. 21 is pivotal for its high $z T$ value, which benefits largely from strong phonon scattering induced by the mass contrast between heavy hafnium and light titanium. Further optimization of material composition can be realized through doping and substitution to reach a compromise between cost and materials performance (e.g., see Ref. 7).

Figure 3(a) shows the experimentally measured current-voltage and power-voltage characteristics of a thermoelectric module with 18 p-type FeNbSb and $18 n$-type TiNiSn legs measured on the test bench imposing a constant hot-side temperature of $605^{\circ} \mathrm{C}$ and a cold-side temperature of $30^{\circ} \mathrm{C}$. Under these conditions, the module generates an open-circuit voltage of $1.9 \mathrm{~V}$ and a short-circuit current of $6.6 \mathrm{~A}$ (corresponding to a high current density in the legs of $215 \mathrm{~A} / \mathrm{cm}^{2}$ ). Matching the external load resistance to the internal resistance of the module, a maximum output power of $3.1 \mathrm{~W}$ is measured experimentally (red line).

The blue line represents simulated values from the steady-state multiphysics model based on heat conservation [Eq. (1) in Ref. 23] and charge conservation [Eq. (2) in Ref. 23] taking the materials data in Fig. 2 and the exact module geometry in Fig. S2 in the supplementary material into account,

$$
-\nabla \cdot(\kappa \nabla T)=\frac{j^{2}}{\sigma}-\nabla \cdot(S T \sigma(\nabla V+S \nabla T))
$$

$$
\nabla \cdot j=0
$$

Here, $T(\mathrm{~K})$ is the local temperature, $j\left(\mathrm{~A} / \mathrm{m}^{2}\right)$ is the local current density, $\rho\left(\mathrm{kg} / \mathrm{m}^{3}\right)$ is the gravimetric density of the material at a specific location, $C_{p}(\mathrm{~J} / \mathrm{kg} \mathrm{K})$ is the specific heat capacity, $\kappa(\mathrm{W} / \mathrm{m} \mathrm{K})$ is the thermal conductivity, $\sigma(\mathrm{S} / \mathrm{m})$ is the electronic conductivity, and $S(\mathrm{~V} / \mathrm{K})$ is the Seebeck coefficient. The model neglecting the radiative heat losses causes a slight deviation between experimental and simulated data. Figure 3(b) shows the results of an extended model based on Eq. (3) in which radiative heat losses are taken into account following the Stefan-Boltzmann law, where the Stefan-Boltzmann constant $\sigma$ SB (not to be confused with electronic conductivity $\sigma$ ) is scaled by an empirical emissivity factor $\varepsilon$, taking into account heat emitted by one leg and absorbed by a neighboring leg including reflection and reemission phenomena,

$$
-\nabla \cdot(\kappa \nabla T)=\frac{j^{2}}{\sigma}-\nabla \cdot\left(S T \sigma(\nabla V+S \nabla T)+\varepsilon \sigma_{S B} T^{4}\right)
$$

The emissivity factor $\varepsilon$ is fixed here at a value of 0.3 , which provides the best match between experiments and model and will be kept constant for all further modeling results, despite the fact that the value may slightly change when the material composition and module geometry are changed.

The model corrected for radiative heat losses can now also be applied to estimate the output performance of a thermoelectric module with best-in-class materials. A comparison of simulated module output data is shown in Fig. 3(c). A hypothetic module with best-in-class Half-Heusler materials would thus generate an open-circuit voltage of $3.4 \mathrm{~V}$, a short-circuit current of $6.4 \mathrm{~A}$, and a maximum output power of $5.4 \mathrm{~W}$. The higher open-circuit voltage 
and consequently higher output power is mainly attributed to the large Seebeck coefficient of the $n$-type material of Ref. 21 .

The model can also be employed to determine the optimum leg dimensions, which maximize module output power under a set of given conditions. In Fig. 3(d), the module area and the number of legs per module were kept fixed at $25 \times 25 \mathrm{~mm}^{2}$ and 36 , respectively, while the impact of leg width $w$ (maintaining a quadratic leg base and constant distance between leg centers) and leg height $h$ on module output power was explored. Inspection of Fig. 3(d) reveals that at fixed cold- and hot-side temperatures $\left(30^{\circ} \mathrm{C}\right.$ and $\left.605^{\circ} \mathrm{C}\right)$, a leg height to leg base area ratio $h / w^{2}$ near $0.5 / \mathrm{mm}$ maximizes output power, with the output power increasing for increasing leg width $w$. If leg height is fixed to $4.0 \mathrm{~mm}$, the optimum leg width is $3.25 \mathrm{~mm}$, resulting in the optimum compromise between minimizing electrical resistance of the module, while maintaining a sufficiently hightemperature gradient across the thermoelectric legs.

While fixed temperature can easily be imposed on a thermoelectric module test bench, leaving it to the heater and cooler to maintain actively constant temperature, the cold- and hot-side heat transfer coefficients $H T C_{c}$ and $H T C_{\mathrm{h}}$ between the thermoelectric module and the heat sink and source are fixed in realistic applications [schematically depicted in Fig. 4(a)]. Under these conditions, cold- and hot-side temperatures depend on module geometry, module material properties, and the temperature of the heat sink and source.
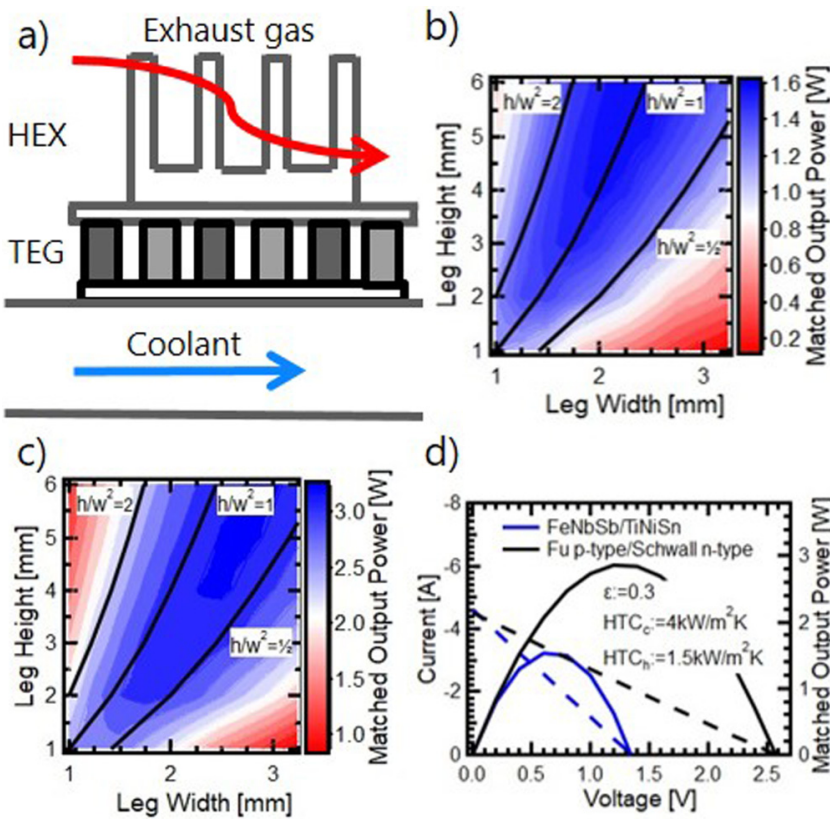

d)

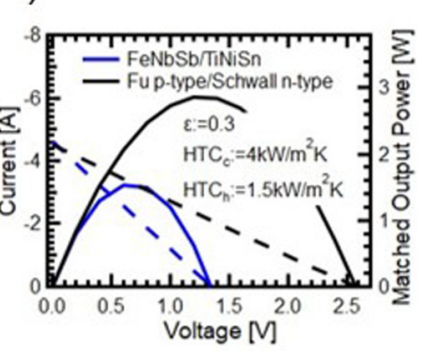

FIG. 4. (a) Schematics showing a thermoelectric module sandwiches between a water-cooled cold-side heat exchanger and a hot-side exhaust gas heat exchanger with lamellar fins, (b) module output power as a function of leg width and leg height for fixed heat transfer coefficient values of the cold and hot-side heat exchanger for a FeNbSb and TiNiSn Half-Heusler module, (c) module output power for best-in-class Half-Heusler materials from Refs. 13 and 21, and (d) comparison of current-voltage and power-voltage characteristics of the module geometries matched (to hot-side) heat exchanger.
For the calculations, we assume a cold-side heat transfer coefficient $\mathrm{HTC}_{\mathrm{c}}$ of $4 \mathrm{~kW} / \mathrm{m}^{2} \mathrm{~K}$ corresponding to a flat watercooled copper plate. Hot-side heat transfer coefficient $\mathrm{HTC}_{\mathrm{h}}$ is set to $1.5 \mathrm{~kW} / \mathrm{m}^{2} \mathrm{~K}$, which corresponds to a realistic value for a copper-based exhaust gas heat exchanger with optimized lamellar fins (five fins per module, with length $30 \mathrm{~mm}$, height $18 \mathrm{~mm}$ ) extracting heat from, e.g., an internal combustion engine's exhaust gas without generating significant backpressure for the charge exchange of the internal combustion engine (while the module test bench provides heat at $140 \mathrm{~kW} / \mathrm{m}^{2} \mathrm{~K}$ to maintain a hot-side temperature of $605^{\circ} \mathrm{C}$ in the previous case). Figure 4(b) shows that under these boundary conditions, a leg height to leg base area ratio $h / w^{2}$ near $1 / \mathrm{mm}$ provides maximum output power for modules with our Half-Heusler materials. If leg height is fixed to $4.0 \mathrm{~mm}$, the optimum leg width is $1.75 \mathrm{~mm}$ (resulting in a temperature difference of $345^{\circ} \mathrm{C}$ ), which differs from the previous fixedtemperature boundary conditions. For the best-in-class materials combination, the maximum shifts to lower $h / w^{2}$ ratios as can be seen in Fig. 4(c), so if keeping a leg height of $4.0 \mathrm{~mm}$, the leg width can be increased to $2.5 \mathrm{~mm}$ to maximize output power. This smaller $h / w^{2}$ ratio results from the different thermoelectric material properties of the materials in Refs. 13 and 21. The significantly lower thermal conductivity and the higher Seebeck coefficient of the best-in-class materials overcompensate the lower electrical conductivity. The ultimate design of a waste-heat-recovery system will also consider the cost of the various components. ${ }^{24}$ For cases in which material costs dominate, it is beneficial to choose a design with lower areal leg coverage and shorter legs. However, for low cost materials, the cost associated with the heat exchanger typically dominates.

Figure 4(d) shows the output power expected from the simulation for an optimized module design with a fixed leg height of $4.0 \mathrm{~mm}$ resulting in an optimum leg width of $1.75 \mathrm{~mm}$ for our materials reaching a maximum output power of $1.6 \mathrm{~W}$. For the same module geometry, the module with the best-in-class materials reaches $2.8 \mathrm{~W}$. These values are lower than $3.1 \mathrm{~W}$ and $5.4 \mathrm{~W}$ obtained at fixed temperatures.

Finally, we integrated the module with our material into a heat exchanger with above specifications mounted to the exhaust of a

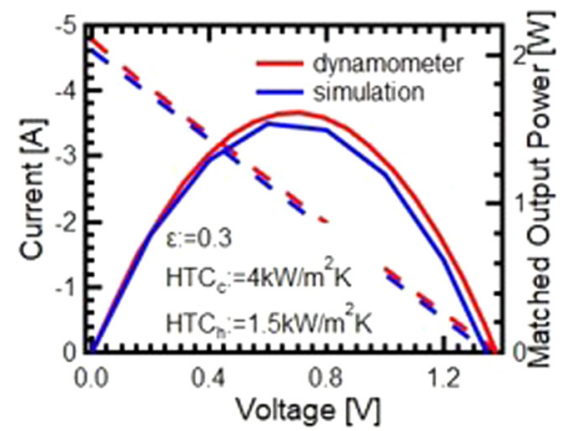

FIG. 5. Comparison of experimental and simulated module output data for a module integrated into a heat exchanger mounted on the exhaust gas conduct of a compact van driving at $100 \mathrm{~km} / \mathrm{h}$. 
compact van with a natural-gas-powered internal combustion engine. Current-voltage and power-voltage characteristics were measured during steady-state operation at $100 \mathrm{~km} / \mathrm{h}$ on a dynamometer. The experimental results show excellent agreement with the simulated results under fixed HTC boundary conditions (Fig. 5).

\section{CONCLUSION}

In summary, we described the fabrication and characterization of thermoelectric modules based on Half-Heusler FeNbSb and TiNiSn. While much of the thermoelectric literature characterizes thermoelectric modules at fixed cold- and hot-side temperatures, our results show that it is essential to consider fixed HTC boundary conditions instead for a given heat exchanger configuration. Multiphysics modeling serves as an important guide for optimizing the module geometry. Future work should be expanded to transient operating conditions, e.g., during a complete standardized driving cycle, the worldwide harmonized light vehicles test procedure (WLTP), ${ }^{25}$ or the federal test procedure (FTP-75). ${ }^{26}$ However, the duration of these cycles is only about $30 \mathrm{~min}$ with a cold engine start and demands only low loads from the internal combustion engine. These conditions are highly disadvantageous to demonstrate the potential of thermoelectric waste-heat recovery due to the thermal inertia and low exhaust gas temperatures. Steady-state cycles starting with warmed-up engines and higher loads such as the world harmonized stationary cycle ${ }^{27}$ (WHSC) for heavy-duty vehicles would provide a more advantageous scenario for wasteheat recovery in automobile applications.

\section{SUPPLEMENTARY MATERIAL}

A description of the test bench for the characterization of thermoelectric modules as well as a schematic of the thermoelectric modules including contacting layers is provided as supplementary material.

\section{ACKNOWLEDGMENTS}

D. Landmann, Y. Tang, and C. Battaglia acknowledge the Swiss Federal Office of Energy for funding support under Contract No. SI/501310-01. The help of J. Hu, M. Huber, T. Bütler, and D. Panayotis for mounting the dynamometer experiment as well as E. Lo Schiavo, M. Hauenstein, and C. Rovath for providing the values for the heat transfer coefficients of the heat exchanger are also acknowledged.

\section{REFERENCES}

${ }^{1}$ G. J. Snyder and E. S. Toberer, Nat. Mater. 7, 105 (2008).

${ }^{2}$ J.-F. Li, W.-S. Liu, L.-D. Zhao, and M. Zhou, NPG Asia Mater. 2, 152 (2010).

${ }^{3}$ X. Zhang and L. Zhao, J. Mater. 1, 92 (2015).

${ }^{4}$ Y. Nishino, M. Kato, and S. Asano, Phys. Rev. Lett. 79, 1909 (1997).

${ }^{\mathbf{5}}$ K. Renard, A. Mori, Y. Yamada, S. Tanaka, H. Miyazaki, and Y. Nishino, J. Appl. Phys. 115, 033707 (2016).

${ }^{6}$ P. Gorai, V. Stevanovic, and E. S. Toberer, Nat. Rev. Mater. 2, 1 (2017).

${ }^{7}$ Y. Tang, X. Li, L. H. J. Martin, E. C. Reyes, T. Ivas, C. Leinenbach, S. Anand, M. Peters, G. J. Snyder, and C. Battaglia, Energy Environ. Sci. 11, 311 (2018).

${ }^{8}$ R. Funahashi, Y. Matsumur, H. Tanaka, T. Takeuchi, W. Norimatsu, E. Combe, R. O. Suzuki, Y. Wang, C. Wan, S. Katsuyama, M. Kusunoki, and K. Koumoto, J. Appl. Phys. 112, 073713 (2012).

${ }^{9}$ H. J. Wu, L.-D. Zhao, F. S. Zheng, D. Wu, Y. L. Pei, X. Tong, M. G. Kanatzidis, and J. Q. He, Nat. Commun. 5, 4515 (2014).

${ }^{10}$ J. R. Salvador, J. Yang, H. Wang, and X. Shi, J. Appl. Phys. 107, 043705 (2010).

${ }^{11}$ H. Hazama, M. Matsubara, R. Asahi, and T. Takeuchi, J. Appl. Phys. 110, 063710 (2011).

${ }^{12}$ G. Joshi, R. He, M. Engber, G. Samsonidze, T. Pantha, E. Dahal, K. Dahal, J. Yang, Y. Lan, B. Kozinsky, and Z. Ren, Energy \& Enviromental Science 7, 4070 (2014).

${ }^{13}$ C. Fu, S. Bai, Y. Liu, Y. Tang, L. Cheng, X. Zhao, and T. Zhu, Nature Communications 6, 8144 (2015).

${ }^{14}$ R. He, G. Schierning, and K. Nielsch, Adv. Mater. Technol. 3, 1700256 (2018).

${ }^{15}$ G. P. Meisner, in 2011 Thermoelectrics Application Workshop (2011); available at https://www.energy.gov/sites/prod/files/2014/03/f13/meisner_0.pdf

${ }^{16}$ M. Nesarajah and G. Frey, Appl. Sci. 7, 634 (2017).

${ }^{17}$ K. Bartholomé, B. Balke, D. Zuckermann, M. Köhne, M. Müller, K. Tarantik, and J. König, J. Electron. Mater. 43, 1775 (2014).

${ }^{18}$ J. R. Salvador, J. Y. Cho, Z. Ye, J. E. Moczygemba, A. J. Thompson, J. W. Sharp, J. D. König, R. Maloney, T. Thompson, J. Sakamoto, H. Wang, A. A. Wereszczak, and G. P. Meisner, J. Electron. Mater. 42, 1389 (2013).

${ }^{19}$ M. Mikami, K. Keizo, K. Tetsuya, K. Kubo, and N. Uchiyama, Jpn. J. Appl. Phys. 47, 1512 (2008).

${ }^{20}$ J. D. Koenig, Nat. Energy 3, 259 (2018).

${ }^{21}$ B. Schwall and M. Balke, Phys. Chem. Chem. Phys. 15, 1868 (2013).

${ }^{22}$ G. J. Snyder and T. S. Ursell, Phys. Rev. Lett. 91, 148301 (2003).

${ }^{23}$ X. Hu, H. Takazawa, K. Nagase, M. Ohta, and A. Yamamoto, J. Electron. Mater. 44, 3637 (2015).

${ }^{\mathbf{2 4}}$ K. Y. Shannon, S. LeBlanc, K. E. Goodson, and C. Dames, Energy Environ. Sci. 6, 2561 (2013).

${ }^{25}$ D. Tsokolis, S. Tsiakmakis, A. Dimaratos, G. Fontaras, P. Pistikopoulos, B. Ciuffo, and Z. Samaras, Appl. Energy 179, 1152 (2016).

${ }^{26} \mathrm{P}$. Soltic, M. Weilenmann, and P. Novak, Inter. J. Enviromnent and Pollution 22(3), 259 (2004).

${ }^{27}$ C.-L. Myung, A. Koh, J. Kim, K. Choi, S. Kwon, and S. Park, J. Mechanical Science and Technology 27(12), 3893 (2013). 